07

\title{
Температурное поведение спектров оптического поглощения квантовых точек InP/ZnS
}

\author{
() С.С. Савченко, А.С. Вохминцев, И.А. Вайнштейн
}

Уральский федеральный университет, НОЦ НАНОТЕХ, Екатеринбург

๑E-mail: i.a.weinstein@urfu.ru

Поступило в Редакцию 7 октября 2016 г.

Проведено исследование спектров оптического поглощения квантовых точек ядро/оболочка InP/ZnS в широком диапазоне температур $T=6.5-296 \mathrm{~K}$. Методом производной спектрофотометрии второго порядка определены энергии оптических переходов при комнатной температуре: $E_{1}=2.60 \pm 0.02 \mathrm{eV}$ соответствует первому экситонному пику поглощения ядра InP, $E_{2}=4.70 \pm 0.02 \mathrm{eV}$ может быть связана с процессами в оболочке $\mathrm{ZnS}$. Впервые для нанокристаллов $\mathrm{InP} / \mathrm{ZnS}$ выполнена аппроксимация экспериментальной зависимости $E_{1}(T)$ в рамках линейной модели и с помощью выражения Фэна. Показано, что изменение энергии $E_{1}$ с температурой обусловлено взаимодействием экситонов с продольными акустическими фононами $\hbar \omega=15 \mathrm{meV}$.

DOI: $10.21883 /$ PJTF.2017.06.44402.16543

Коллоидные квантовые точки (КТ) или полупроводниковые нанокристаллы привлекают внимание исследователей в настоящее время благодаря своим уникальным люминесцентным свойствам и возможностям тонкой настройки оптических характеристик при разработке современных светоизлучающих устройств, солнечных элементов и др. [1-6]. КТ на основе соединений III-V групп представляют особый интерес, поскольку не содержат токсичных элементов $(\mathrm{Cd}, \mathrm{Pb}, \mathrm{Hg})$ и могут широко применяться, в том числе для создания биосенсоров, биомаркеров и различных биосовместимых приложений [7-9]. С этой точки зрения одной из перспективных функциональных сред являются нанокристаллы фосфида индия, покрытые оболочкой из сульфида цинка [10]. Известно, что ширину запрещенной зоны в InP/ZnS, которая определяется положением первого экситонного пика в спектрах оптического поглощения, можно варьировать в широких пределах за счет изменения размера 
КТ [11]. Другим фактором, влияющим на эту величину, является температура. При этом анализ температурной зависимости позволяет получать фундаментальную количественную информацию о параметрах электрон-фононного взаимодействия. В настоящее время для полупроводниковых нанокристаллов $\mathrm{InP} / \mathrm{ZnS}$ известны исследования температурного изменения спектров фотолюминесценции [12-14]. В данной работе проанализировано поведение спектров оптического поглощения KТ ядро/оболочка InP/ZnS в температурном интервале 6.5-296 K.

Исследуемые КT InP/ZnS (производитель ФГУП „НИИ прикладной акустики“, Дубна) имеют трехслойную структуру: ядро из InP, оболочка из $\mathrm{ZnS}$ и покрытие из модифицированной полиакриловой кислоты. Исходные образцы нанокристаллов представляли собой водный коллоидный раствор с концентрацией $C=40 \mathrm{~g} / 1$, характеризовались полосой эмиссии с максимумом $513 \mathrm{~nm}$ и полушириной $40 \mathrm{~nm}$, квантовый выход люминесценции по данным изготовителя составлял 13\%. Путем последовательного разбавления дистиллированной водой была приготовлена концентрационная серия в диапазоне $C=40-0.04 \mathrm{~g} / 1$. Измерения спектров оптического поглощения (ОП) коллоидных растворов с различной концентрацией КТ были выполнены в кварцевой кювете с длиной оптического пути $1 \mathrm{~cm}$ при комнатной температуре с использованием спектрофотометра Shimadzu UV-2450. Стабильность полученных растворов была подтверждена линейной концентрационной зависимостью измеренной оптической плотности $(A)$ для длин волн 260, 370 и $470 \mathrm{~nm}$.

Изучение температурной зависимости поглощения нанокристаллов было выполнено для сухого осадка InP/ZnS. Он формировался путем физического осаждения коллоидного раствора исходной концентрации на кварцевую подложку толщиной $1 \mathrm{~mm}$ при комнатной температуре. Измерения спектров ОП при $T=6.5-296 \mathrm{~K}$ проводились с помощью установки на базе спектрофотометра Shimadzu UV-2450 и гелиевого криостата Janis модели CCS-100/204N с замкнутым контуром и сенсором температуры DT-670B-CU. Вакуум внутри криостата $\left(7 \cdot 10^{-5} \mathrm{mbar}\right)$ создавался с использованием турбонасосной станции HiCube 80 Eco. Управление температурой и термостатирование осуществлялось посредством контроллера Model 335. Спектры ОП регистрировались при фиксированных температурах: $6.5 \mathrm{~K}$, в диапазоне $10-100 \mathrm{~K}$ с шагом $10 \mathrm{~K}$ и в диапазоне $100-296 \mathrm{~K}$ с шагом $20 \mathrm{~K}$. В ходе измерений спектральная ширина щели составляла $2 \mathrm{~nm}$, интервал сканирования $0.5 \mathrm{~nm}$. 

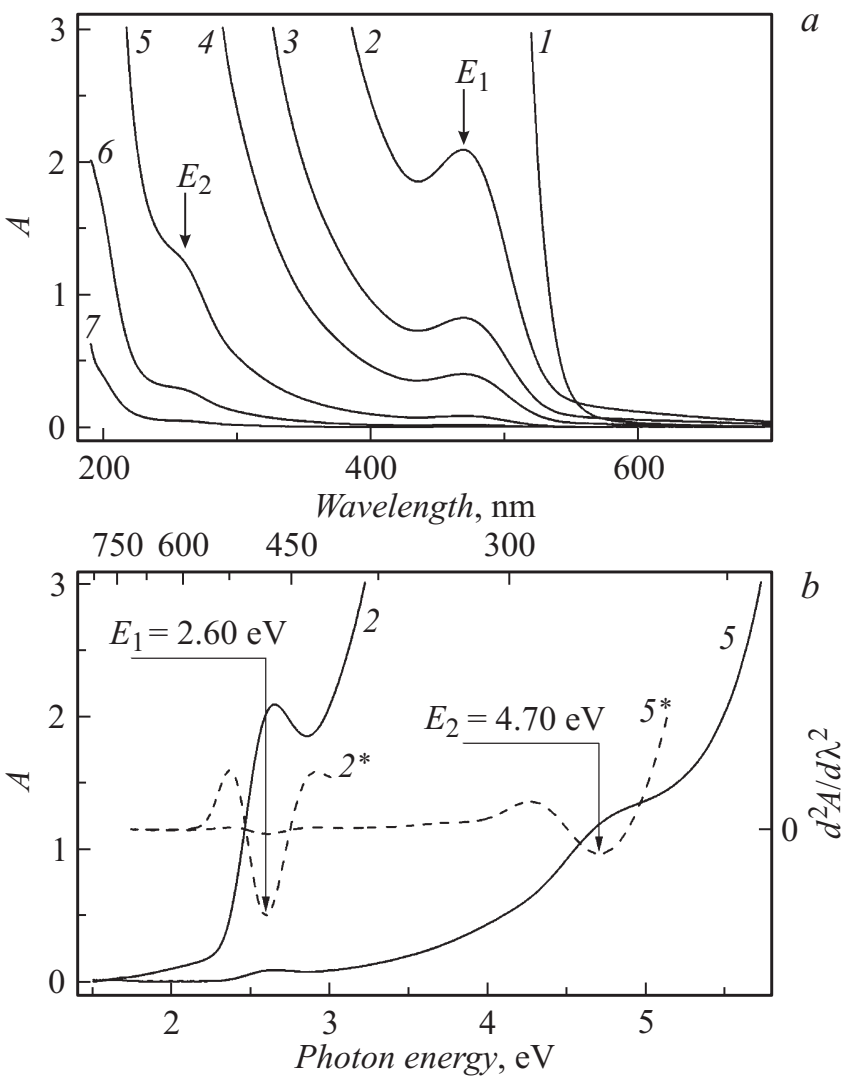

Рис. 1. Спектры ОП коллоидных растворов $\mathrm{InP} / \mathrm{ZnS}: a$ - для различных концентраций, g/l: $1-40,2-10,3-5,4-2.5,5-0.63,6-0.16,7-$ $0.04 ; b-$ примеры спектров второй производной ОП (штриховые кривые $2^{*}$ и $5^{*}$ ) для соответствующих концентраций.

На рис. 1, $a$ представлены спектры ОП для исследуемой серии растворов КТ. Видно, что с уменьшением концентрации InP/ZnS величина $A$ монотонно падает, позволяя наблюдать особенности поглощения в широком спектральном диапазоне $200-700 \mathrm{~nm}$. При этом для кривых 2-4 наблюдается пик $E_{1}$ в диапазоне $480-450 \mathrm{~nm}$, а для кривых 5-7

Письма в ЖТФ, 2017, том 43, вып. 6 
обнаруживается плечо $E_{2}$ в интервале $270-240 \mathrm{~nm}$. Отметим, что, в отличие от идеализированного теоретического описания, поглощение реальных КТ проявляет себя в виде максимумов и/или плечей на некотором монотонно возрастающем фоне (предположительно, вклад соответствующей объемной плотности состояний, процессов рассеяния или др. $[3,11])$. В этой связи для характеризации оптических переходов и определения положений скрытых полос поглощения могут быть использованы подходы производной спектрофотометрии $[15,16]$.

В настоящей работе все экспериментально полученные спектры ОП нанокристаллов были проанализированы на основе применения метода второй производной (рис. $1, b)$. Оценки энергий оптических переходов, которые были сделаны по положению минимумов $d^{2} A / d \lambda^{2}$, показаны стрелками и составляют $E_{1}=2.60 \pm 0.02 \mathrm{eV}$ и $E_{2}=4.70 \pm 0.02 \mathrm{eV}$. Отметим, что рассчитанные значения не меняются для коллоидных растворов с разной концентрацией КТ, что является дополнительным подтверждением стабильности исследуемого ряда.

Согласно литературным данным, пик $E_{1}$ может быть приписан первой экситонной полосе поглощения фосфида индия. Его энергия существенно сдвинута относительно величины ширины запрещенной зоны объемного InP $(1.34 \mathrm{eV}$ при $T=290 \mathrm{~K}[17])$ в силу проявления эффекта квантового ограничения. Значение $E_{2}$ попадает в интервал энергий 4.47-4.82 eV, которые были получены для ширины запрещенной зоны нанокристаллических порошков сульфида цинка в [18]. Таким образом, указанный оптический переход может быть отнесен к оболочке $\mathrm{ZnS}$. Отметим, что детальный анализ данного вопроса требует дальнейших исследований, которые выходят за рамки настоящей работы.

На рис. 2, а представлены спектры ОП InP/ZnS, измеренные при различных температурах. Можно видеть, что при охлаждении происходит сдвиг первой экситонной полосы поглощения в сторону более высоких энергий и наблюдается рост соответствующей оптической плотности. Вставка на рисунке более детально иллюстрирует эти изменения. Для определения энергии $E_{1}$ при различных температурах также использовался метод второй производной (рис. 2, $b$ ). Полученные значения представлены на рис. 3. Можно видеть, что $E_{1}(T)$ растет с уменьшением температуры и демонстрирует поведение, характерное для температурно-индуцированного изменения ширины оптической щели в объемных полупроводниковых кристаллах $[17,19]$.

Письма в ЖТФ, 2017, том 43, вып. 6 


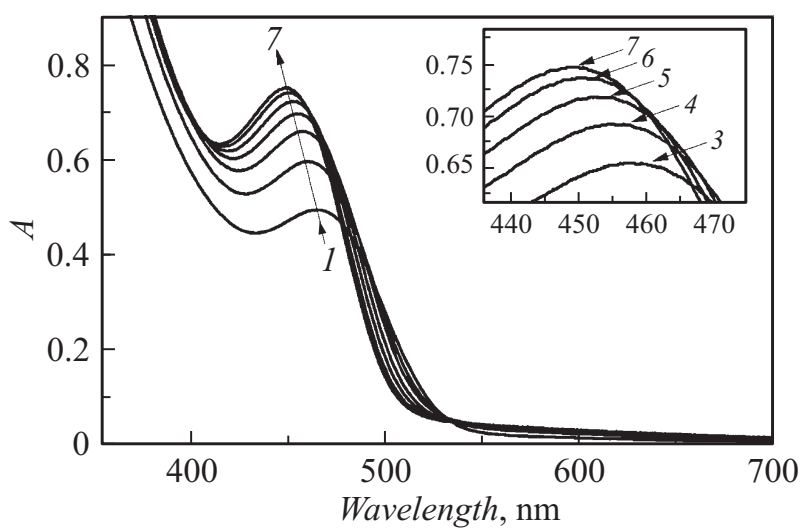

\section{$a$}

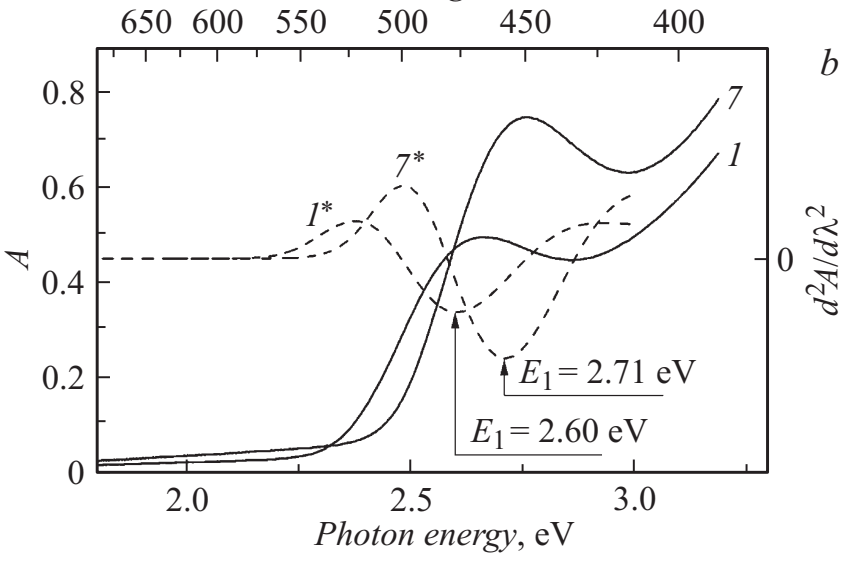

Рис. 2. Спектры ОП осажденных нанокристаллов InP/ZnS: $a$ - измеренные при различных температурах, К: 1 - 296, 2 - 240, 3 - 200, 4 - 160, 5 - 120, $6-80,7-6.5 ; b-$ примеры спектров второй производной ОП (штриховые кривые $1^{*}$ и $7^{*}$ ) для соответствующих температур.

В настоящей работе для количественного анализа экспериментальных зависимостей $E_{1}(T)$ использовались выражение Фэна для температурного поведения ширины запрещенной зоны в кристаллах [19]

$$
E_{1}(T)=E_{1}(0)-A_{\mathrm{F}}\left[\exp \left(\frac{\hbar \omega}{k T}\right)-1\right]^{-1},
$$

Письма в ЖТФ, 2017, том 43, вып. 6 


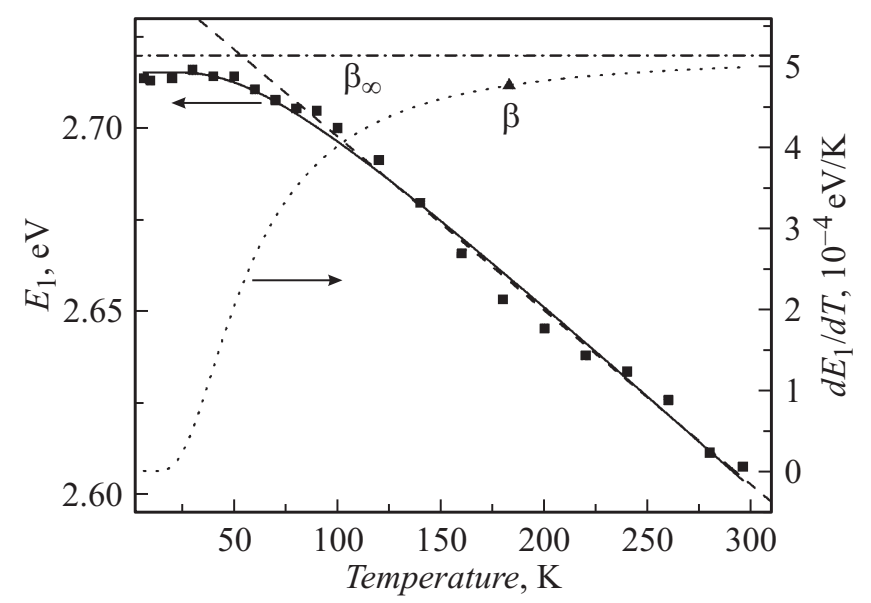

Рис. 3. Температурная зависимость энергии $E_{1}(T)$ для $\mathrm{InP} / \mathrm{ZnS}$ : квадратные символы - экспериментальные оценки; сплошная линия - аппроксимация по выражению Фэна (1); штриховая линия - аппроксимация в рамках линейной модели (2); пунктирная линия - первая производная функции $E_{1}(T)$; штрихпунктирная линия - уровень $\beta_{\infty}$; треугольный символ - значение температурного коэффициента $\beta$, рассчитанное согласно выражению (2).

а также полуэмпирическая линейная модель

$$
E_{1}(T)=E_{1}(0)-\beta T .
$$

Здесь $E_{1}(0)$ - энергия оптического перехода при $0 \mathrm{~K}, \mathrm{eV} ; A_{\mathrm{F}}-$ параметр Фэна, зависящий от микроскопических свойств материала $[19,20], \mathrm{eV}$; выражение в квадратных скобках в правой части (1) фактор Бозе-Эйнштейна для фононов с энергией $\hbar \omega ; k-$ постоянная Больцмана, $\mathrm{eV} / \mathrm{K} ; \beta$ - температурный коэффициент смещения энергетических уровней (аналог температурного коэффициента ширины запрещенной зоны в объемных материалах), eV/K.

Ранее в [19] было показано, что в пределе высоких температур $(k T \gg \hbar \omega)$ соотношение (1) сводится к виду (2), при этом температурный коэффициент записывается как

$$
\beta_{\infty}=A_{\mathrm{F}} \frac{k}{\hbar \omega} .
$$

Письма в ЖТФ, 2017, том 43, вып. 6 
Параметры температурной зависимости для первого экситонного пика поглощения $\mathrm{InP} / \mathrm{ZnS}$

\begin{tabular}{c|c|c|c|c}
\hline Нанокристаллы & $E_{1}(0), \mathrm{eV}$ & $A_{\mathrm{F}}, \mathrm{eV}$ & $\hbar \omega, \mathrm{meV}$ & $\beta, 10^{-4} \mathrm{eV} / \mathrm{K}$ \\
\hline \multirow{2}{*}{ KT InP/ZnS } & $2.715 \pm 0.002$ & $0.089 \pm 0.016$ & $\begin{array}{c}15 \pm 2 \\
13[12]\end{array}$ & $4.76 \pm 0.16$ \\
& & & $23[13]$ & \\
\hline Объемный InP & $1.42[17]$ & $0.050[19]$ & 15.5 (LA) [21] & $4.6[17]$
\end{tabular}

Исследование температурной зависимости ОП материалов с использованием выражения (1) позволяет получать количественную информацию об эффективной энергии фононов, электронно-колебательные взаимодействия с которыми в значительной степени определяют смещение энергетических уровней. На рис. 3 показана аппроксимация экспериментальной зависимости $E_{1}(T)$ с использованием соотношения Фэна (сплошная линия) и линейной модели (штриховая линия). Полученные при аппроксимации параметры в сравнении с независимыми литературными данными представлены в таблице. Видно, что расчетное значение $\hbar \omega$ соответствует энергии продольных акустических фононов в объемном InP и согласуется с результатами, полученными для КТ в $[12,13]$. Следует отметить, что в указанных работах были изучены температурные зависимости фотолюминесценции нанокристаллов $\mathrm{InP} / \mathrm{ZnS}$ различных размеров в диапазоне $2-510 \mathrm{~K}$.

На рис. 3 показана также зависимость величины $d E_{1} / d T$ (пунктирная линия), которая характеризует изменение температурного коэффициента $\beta$. Штрихпунктирная линия на рис. 3 соответствует уровню $\beta_{\infty}=5.13 \cdot 10^{-4} \mathrm{eV} / \mathrm{K}$, вычисленному по выражению (3). Значение $\beta=4.76 \cdot 10^{-4} \mathrm{eV} / \mathrm{K}$, показанное темным треугольником на рис. 3 , было рассчитано на основе аппроксимации $E_{1}(T)$ в диапазоне $90-296 \mathrm{~K}$ по выражению (2) (штриховая линия на рис. 3) и соответствует справочным данным для объемного InP [17] (см. таблицу). В общем случае значение $\beta$ будет зависеть от интервала $T$, используемого для линейной аппроксимации. Поскольку в настоящей работе выполняется соотношение $\beta \approx \beta_{\infty}$, можно говорить, что рассматриваемый интервал 90-296 К для исследуемых КТ вполне удовлетворяет высокотемпературному условию $k T \gg \hbar \omega$.

Письма в ЖТФ, 2017, том 43, вып. 6 
Таким образом, в работе исследованы спектры оптического поглощения коллоидных КT InP/ZnS в диапазоне концентраций от 40 до $0.04 \mathrm{~g} / 1$ при комнатной температуре. С помощью метода производной спектрофотометрии второго порядка проведена оценка энергий оптических переходов, которые составляют $E_{1}=2.60 \pm 0.02 \mathrm{eV}$, $E_{2}=4.70 \pm 0.02 \mathrm{eV}$ и не зависят от концентрации растворов КТ. Величина $E_{1}$ соответствует первому экситонному пику поглощения ядра InP, а $E_{2}$ может быть приписана переходам в оболочке $\mathrm{ZnS}$. Впервые проанализирована температурная зависимость энергии $E_{1}$ для $T=6.5-296 \mathrm{~K}$. Температурный коэффициент $\beta=4.76 \cdot 10^{-4} \mathrm{eV} / \mathrm{K}$ в интервале $T=90-296$ К соответствует значению аналогичного параметра для объемных кристаллов InP. Обнаружено, что изменение положения первого экситонного пика поглощения с температурой обусловлено главным образом взаимодействием экситонов с продольными акустическими фононами $\hbar \omega=15 \mathrm{meV}$.

Исследование выполнено при содействии программы 211 правительства РФ (соглашение № 02.А03.21.0006). А.С. Вохминцев благодарит за поддержку грант Президента РФ № МK-5729.2015.2.

\section{Список литературы}

[1] Zhang Q., Wang C-F., Ling L-T., Chen S. // J. Mater. Chem. C. 2014. V. 2. P. 4358.

[2] Klimov V.I., Mikhailovsky A.A., Xu S. et al. // Science. 2000. V. 290. P. 314.

[3] Weller H. // Angew. Chem. (Int. Ed. Engl.) 1993. V. 32. P. 41.

[4] Rempel A.A., Kozlova E.A., Gorbunova T.I. et al. // Catal. Commun. 2015. V. 68. P. 61.

[5] Ремпель А.А. // Известия АН. Сер. химическая. 2013. Т. 62. С. 857.

[6] Gaponenko S.V. Optical Properties of Semiconductor Nanocrystals. Cambridge: Cambridge University Press, 1998. 245 p.

[7] Kamat P.V. // J. Phys. Chem. C. 2008. V. 112. P. 18737.

[8] Savchenko S.S., Vokhmintsev A.S., Weinstein I.A. // AIP Conf. Proc. 2016. V. 1717. P. 040028.

[9] Chatterjee K., Sarkar S., Jagajjanani Rao K., Paria S. // Adv. Colloid Interface Sci. 2014. V. 209. P. 8.

[10] Бричкин С.Б., Спирин М.Г., Товстун С.А. и др. // ХВЭ. 2016. Т. 50. С. 417.

[11] Reiss P., Protiere M., Li L. // Small. 2009. V. 5. P. 154.

Письма в ЖТФ, 2017, том 43, вып. 6 
[12] Narayanaswamy A., Feiner L.F., Van Der Zaag P.J. // J. Phys. Chem. C. 2008. V. 112. P. 6775.

[13] Narayanaswamy A., Feiner L.F., Meijerink A., Van Der Zaag P.J. // ACS Nano. 2009. V. 9. P. 2539.

[14] Pham T.T., Tran T.K.C., Nguyen Q.L. // Adv. Nat. Sci. Nanosci. Nanotechnol. 2011. V. 2. P. 025001.

[15] Бёккер Ю. Спектроскопия. М.: Техносфера. 2009. 528 с.

[16] Talsky G. Derivative Spectrophotometry: Low and Higher Order. Weinheim: $\mathrm{VCH}, 1994.229 \mathrm{p}$.

[17] Бабичев А.П. Физические величины: Справочник / Под. ред. И.С. Григорьева, Е.3. Мейлихова. М.: Энергоатомиздат, 1991. 1232 с.

[18] Kho R., Torres-Martınez C.L., Mehra R.K. // J. Colloid Interface Sci. 2000. V. 227. P. 561.

[19] Вайнштейн И.А., Зацепин А.Ф., Кортов В.С. //ФТТ. 1999. Т. 41. С. 994.

[20] Fan H.Y. // Phys. Rev. 1951. V. 82. P. 900. Alfrey G.F., Borcherds P.H. // J. Phys. C: Solid State Phys. 1972. V. 20. P. L275.

[21] Alfrey G.F., Borcherds P.H. // J. Phys. C: Solid State Phys. 1972. V. 20. P. L275.

Письма в ЖТФ, 2017, том 43, вып. 6 